\section{Absolute beginnings}

\section{Degrees Kelvin: A Tale of Genius,} Invention, and Tragedy

by David Lindley

Joseph Henry Press: 2004. 366 pp. \$27.95

\section{David B. Wilson}

Recovering unfamiliar ideas from the past can be difficult, but David Lindley accepts the challenge, providing for the most part a fine survey of Lord Kelvin's life for a general audience. His narrative takes us from the child prodigy William Thomson to his later years as Lord Kelvin, the most honoured scientist and engineer of Victorian Britain. Lindley's early investigations formed the impression in his mind of a sharp contrast between the youthful Thomson, largely responsible for the science of thermodynamics, and the elderly Kelvin, unreasonably opposed to new scientific theories. Lindley's book explores that contrast.

Born in Belfast in 1824, Kelvin was only ten when he entered the University of Glasgow, where his father was the professor of mathematics. In his mid-teens, Kelvin published a paper correcting errors in a book by a professor of mathematics at the University of Edinburgh. From Glasgow, Kelvin went to the University of Cambridge, graduating with high honours in 1845. Extending work by the likes of Joseph Fourier and Michael Faraday, his early papers concerned heat, electricity, magnetism and light, as he presented mathematical analogies and mechanical models of these phenomena. In part, he was developing a theory of an elastic-solid ether, vibrations of which constituted light.

In 1846 Kelvin became professor of natural philosophy at the University of Glasgow. In the early 1850 s, he and Rudolf Clausius established the modern science of thermodynamics. In the 1850s and 1860s, he was key to the successful laying of the Atlantic cable, which allowed telegraphic communication between the United States and Britain. In the mid-1860s, Kelvin used thermodynamics to refute the uniformitarian geology of the day, estimating that Earth was much younger than the geologists claimed, and he sought to unite ether and matter with a theory of smoke-ring-like vortex atoms.

Although his early research had inspired James Clerk Maxwell to develop his electromagnetic theory of light, Kelvin resisted Maxwell's conclusions and continued to seek suitable mechanical models of these phenomena. He also resisted darwinian evolution and aspects of the atomic models developed by J. J. Thomson and Ernest Rutherford to explain radioactivity. $\mathrm{He}$ became Lord Kelvin in 1892, resigned his professorship in 1899 and died in 1907.

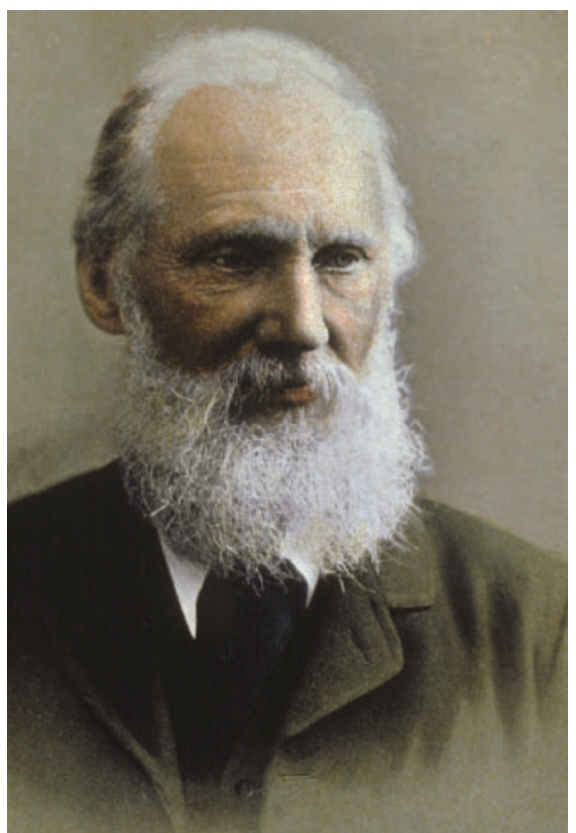

Broad-minded? Lord Kelvin's work ranged from thermodynamics to electromagnetism.

Lindley's story of this remarkable life goes admirably beyond the ideas of science and engineering to reveal much of the dayto-day Kelvin. To this end, Lindley liberally quotes from a substantial family and scientific correspondence. As a Cambridge undergraduate, Kelvin received much fatherly advice about studying hard and behaving properly. The family's advice later turned towards Kelvin's preparation to apply for the Glasgow chair of natural philosophy when a vacancy seemed in the offing - his sister

\section{New in paperback}

Strange Matters: Undiscovered Ideas at the Frontiers of Space and Time by Tom Siegfried

Berkley, $\$ 15$

"Not just a tour of current theoretical speculation on time, space and matter, but rather a cogent argument as to why we should take these exotic ideas seriously." Marc Kamionkowski Nature 420, 362-363 (2002).

Rare Earth: Why Complex Life is Uncommon in the Universe

by Peter D. Ward \& Donald Brownlee Copernicus, £15.50, \$16.95

Small Worlds: The Dynamics of Networks between Order and Randomness by Duncan J. Watts

Princeton University Press, \$24.95, £16.95

Spying with Maps: Surveillance Technologies and the Future of Privacy

by Mark Monmonier

University of Chicago Press, \$16, £11.50 advised Kelvin to grow a beard, to give him authority.

Other correspondence cited by Lindley reveals much of the relations between Kelvin and other scientists. In the early 1840s, Kelvin met G. G. Stokes, a Cambridge honours graduate of 1841, and their extensive correspondence attests to both their friendship and their common scientific interests, although the former seldom appeared explicitly in their letters, as Lindley notes. Another Cambridge honours graduate, P. G. Tait, championed Kelvin's physics, although their collaboration on the monumental Treatise on Natural Philosophy (1867) filled their letters with tales of Kelvin's delays and Tait's exasperation. Then there was longevity's sad side as colleagues died, Tait in 1901 and Stokes in 1903.

Lindley does a splendid job of explaining the scientific and technological concepts for a general audience, but at a deeper level the book falls short. His present-minded perspective too often misrepresents the historical context of ideas. Occasionally, subjects are misleadingly presented as not being well understood until the modern (that is, correct) theory appeared. The MichelsonMorley experiment of 1887 is mistakenly described as an attack on the ether, a view that is not really plausible without the hindsight provided by relativity theory some two decades later.

Applied to Kelvin's later dissents, this hindsight approach portrays him as being too practical and unimaginative to embrace new truths. However, such a judgment overlooks, for example, the significant scientific difficulties confronting natural selection difficulties that caused Darwin to modify his views. Early atomic models faced similar problems, and acceptance of Maxwell's electromagnetic theory was hardly a straightforward matter. Indeed, in the late Victorian context, Kelvin's alternative to the current atomic models agreed with both his estimate of Earth's age and his objections to natural selection, forming a coherent and imaginative world view. Here, hindsight hides imagination.

If Lindley's extensive research insufficiently modified his early impression of Kelvin, his readers will nevertheless find much insight into the life and ideas of one of Victorian Britain's most interesting and influential men. It will certainly not replace Energy and Empire (Cambridge University Press, 1989) by Crosbie Smith and M. Norton Wise as the definitive biography of Kelvin, but at well under half the length it will undoubtedly be more accessible for most readers.

David B. Wilson is professor of history, mechanical engineering and philosophy at Iowa State

University, Ames, Iowa 50011, USA. He is the author of Kelvin and Stokes: A Comparative Study in Victorian Physics. 